\title{
"Close-Relationship" in the Uralian Languages ${ }^{1}$
}

Suppose three related languages $A, B, C$. Let it be given that, of these three, no one is directly descended from one of the other two. Then, clearly, the three languages may be related in four ways:

\section{TABLE I}

(1)

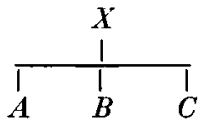

(3)

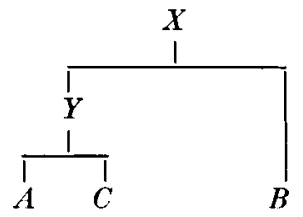

(2)

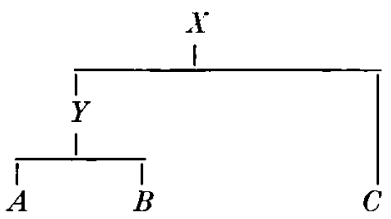

(4)

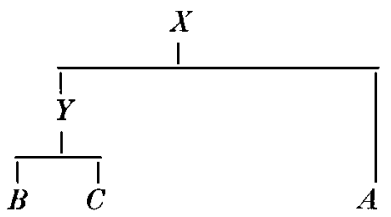

(where $X$ and $Y^{\circ}$ are parent languages). In "Pattern of Descent) No. 2, the relationship between $A$ and $B$ is said to be "closer" than the relationship of $C$ to either $A$ or $B$. It seems to us that nearly all the evidence for assessing the closeness of relationship between two languages of a family of related languages is comprised in a table made up as follows. Consider the cognate words of the languages - that is, words appearing in two or more languages. Allot a column to each language and a row to each cognate word; if a word is present in a language put a cross in the appropriate cell of the table ${ }^{2}$. Thus:

1 Abbreviation: Ross = A. S. C. Ross, "Philological probability problems", Journal of the Royal Statistical Society B. XII. 19-59.

${ }^{2}$ Cf. Ross pp. $26-27$. 
TABLE II

Word

Number
1
2
3

$\begin{array}{ccc}1 & 2 & \\ \times & \times & \\ & \times & \times \\ \times & \times & \times\end{array}$

In the present study we attempt to investigate close-relationship in the Uralian family of languages, using an inventory of words of the type referred to above. The inventory was taken from B. Collinder, Fenno-ugric vocabulary. And, in this context, we should observe, first, that, although it may well be that this source is not perfect, it is nevertheless the only one which is conveniently available. Secondly, if there are omissions in it, as is quite possible, then our conclusion will have been based on only a part of the evidence; nevertheless, this incomplete evidence - if incomplete it be - will suffice for all the conclusions drawn.

Our interest then lies in the question of how close-relationships can be discovered by using quantitative measures of association between languages based on the whole inventory. The evolution of a family of languages is undoubtedly a complex process. As indicated above, we have adopted the junggrammatisch model; that is, we suppose that relationship between a pair of languages implies the existence at some time past of a common ancestor or parent language. The problem is then to construct the family tree of all languages of the family and their lines of descent from their parent languages.

In Glottochronology, a further aim is to attach a time-scale to the lines of descent from the parent language. The construction of a theory to achieve this involves some strict, and generally implausible assumptions as to constant rates of disappearance of words from languages over time. If we consider a language from time $\tau=t$ to $\tau=T$, then it is certainly true to say that the $i$ th of its words existing at $\tau=t$ has a chance $p_{i \tau}$ of dying out in the interval $\tau, \tau+\Delta \tau$. The basic attitude of the glottochronologists may be expressed by supposing them to say that all, or, at worst, whole groups of these probabilities are the same. The basic attitude of their opponents is, 
essentially, to deny this; some of them would go so far as to say that $p_{i \tau}$ has a different value for every $i, \tau$, or at the least for every $i$. In other words, that the chance of a word dying out at a particular time has something to do with the word and the time themselves, or at the least with the word itself. It may also be observed that, in the majority of cases in which Glottochronology has been used in practice, there has been a total absence of historical evidence whereby its results can be checked. So we may conclude this section by emphasising that no attempt will here be made to consider time as a factor in relation to family trees of languages.

We now make some general observations on measures of association between related languages and their use in constructing family trees.

\section{Data and notation}

It is supposed here that the available data consists of an inventory of $N$ words by means of which it is appropriate to investigate the associations of a group of $l$ languages. Suppose the languages are labelled $L_{1}, I_{2} \ldots L_{l}$. Most of the work on association measures between languages is based on computing certain quantities. For any pair of languages $L_{i}$ and $L_{j}$, the total numbers of inventory words present in $L_{i}$ and $L_{j}, n_{i}$ and $n_{j}$ respectively, are calculated. The number of cases in which a word is present in both $L_{i}$ and $L_{j}, r_{i j}$, is also obtained. With the aid of a computer, it would be possible to proceed further and calculate the number of words common to groups of three or more of the languages. Though these quantities would undoubtedly contain information about the association between languages of the family, it will be seen that the difficulty lies in knowing how to exploit or indeed extract this information, particularly when the number of languages is large. Our work will be confined to the use of the $r_{i j}$ only.

Measures of association between language pairs

It has been argued by a number of authors (for example, Ross) that a measure of association should depend only on 
the number of words in common, $r_{i j}$, and not on the number of words absent in both languages; this can be seen to be $N-n_{i}$ $--n_{j}+r_{i j}$. Some examples of measures of this type are the following.

(I) A. Ellegård, "Statistical measurement of linguistic relationship", Language $\mathrm{XXXV}, 131-56$, proposed $c_{i j}$, the ratio of $r_{i j}$ to the geometric mean of the "richnesses» of the languages, $n_{i}$ and $n_{j}$, as an association coefficient;

$$
c_{i j}=r_{i j} /\left(n_{i} n_{j}\right)^{1 / 2} \text {. }
$$

(II) A. Henrici, "Numerical classification of Bantu languages", African language studies XIV, 82-104, used $c^{\prime}{ }_{i j}$, the ratio of $r_{i j}$ to the arithmetic mean of the richnesses;

$$
c^{\prime}{ }_{i j}=r_{i j} / \frac{1}{2}\left(n_{i}+n_{j}\right) \text {. }
$$

(III) A similar measure to Henrici's is $s$ - which will be here referred to as the "similarity coefficient" - the proportion of words common to both languages relative to the proportion of words in either language;

$$
s_{i j}=r_{i j} /\left(n_{i}+n_{j}-r_{i j}\right) .
$$

Neither the inventory size, $N$, nor the numbers of words absent in pairs of languages enters explicitly into these measures. Further, since all the measures are proportional to $r_{i j}$, it is to be expected that, whichever of the three measures is used, comparisons between languages should give similar results. Henrici reports that this is true in practice.

Various authors have made attempts to find ways of studying language associations more sophisticated than looking at simple measures of association. We now briefly describe some of these methods.

Statistical measures of association

If a pair of languages had evolved from different parent languages without interborrowing of words, it is nevertheless conceivable that the same word should occur in both languages 
purely by chance. A probability model for such a chance mechanism is the following - the argument is conditional upon the richnesses $n_{i}$ and $n_{j}$ of languages $L_{i}$ and $L_{j}$ being treated as fixed. Suppose that the $n_{j}$ words of $L_{j}$ are randomly sampled from the $N$ inventory words, and that the count, $r_{i j}$, of those words also present in $L_{i}$ has been made. Then it is well known (cf. for example Ross p. 27) that $r_{i j}$ has a hypergeometric distribution with

$$
\begin{gathered}
\text { Mean }=E\left(r_{i j}\right)=n_{i} n_{j} / N ; \\
\text { and Variance }=\operatorname{Var}\left(r_{i j}\right)=\frac{n_{i} n_{j}}{N-1}\left(1-n_{i} / N\right)\left(1-n_{j} / N\right) .
\end{gathered}
$$

Further, if $n_{i}$ and $n_{j}$ are large, as they usually are for language inventories, the standardised statistic

$$
z_{i j}=\frac{r_{i j}-n_{i} n_{j} / N}{\frac{n_{i} n_{j}}{N-1}\left[\left(1-n_{i} / N\right)\left(1-n_{j} / N\right)\right]^{1 / 2}}
$$

has approximately the Normal distribution with zero mean and unit variance.

We may then apply a significance test to reject the hypothesis of no association. This hypothesis would be rejected at probability level $p$, where $p$ is the probability of obtaining a value of $z_{i j}$ greater than or equal to the observed value. That is

$$
p=\int_{z_{i j}}^{\infty} \frac{1}{\sqrt{2 \pi}} e^{-1 / 2 t^{2}} d t
$$

As there is a strict monotonic decreasing relationship between $z_{i j}$ and $p$, either the positive size of $z_{i j}$ or the smallness of $p$, is a measure of association between the languages. Usually it is more convenient to work with $z_{i j}$, but, for future reference, we give here a short table of the correspondence-values of $p$ and $z_{i j}$.

At this point it is to be noted that this association measure does depend implicitly on the number of words absent in both languages. As it depends on all four of $r_{i j}, n_{i}, n_{j}$ and $N$, it is 
TABLE II

$\begin{array}{lc}z_{i j} & p \\ 2.00 & 0.022750100 \\ 3.00 & 0.001349900 \\ 1.00 & 0.000031700 \\ 5.00 & 0.00000028 \\ 6.00 & 0.000000001\end{array}$

possible to write it in terms of the number of words absent in both, $N-n_{i}-n_{j}+r_{i j}$. However, from the point of view of statistical significance, it is surely right to consider the size of the inventory $N$, and so this fact cannot be held a serious disadvantage.

Constant survival rate model for separation of languayes

There are some points of connection with another measure of relationship proposed by D. G. Kendall (Ross pp. 41--42). Suppose that, at some point of time, languages $L_{i}$ and $L_{j}$ had a common parent consisting of $S_{i j}$ words. Suppose $a_{i}$ and $a_{j}$ are the probabilities of survival for any word along the lines of descent to $L_{i}$ and $L_{f}$ respectively. It has to be assumed that the probability is the same for all words, which is almost certainly not true (cf. p. 26 above), but, if $\alpha_{i}$ and $\alpha_{j}$ are interpreted as average probabilities, the model may be reasonably adequate. It is also assumed that the extinction of any one word is independent of that of any other. Then it can be shown that appropriate estimates of $S_{i j}, a_{i}$ and $a_{j}$ are

$$
S_{i j}=\frac{n_{i} n_{j}}{r_{i j}}, \quad \alpha_{i}=\frac{r_{i j}}{n_{j}}, \quad \alpha_{j}=\frac{r_{i j}}{n_{i}} .
$$

Such estimates would be obtained, for instance, by applying the well-known statistical principle of the method of moments to the problem.

Clearly the quantity $S_{i j}$ will be large when $r_{i j}$ is small, a state of affairs which would perhaps imply an early separation of the languages. 
We see, too, that $S_{i j}$ can be related to the numerator of the $z_{i j}$-statistic, $r_{i j}-n_{i} n_{j} / N$, for we can write

$$
r_{i j}-n_{i} n_{j} / N=n_{i} n_{j}\left(\frac{1}{S_{i j}}-\frac{1}{N}\right) \text {. }
$$

As the denominator of the $z_{i j}$-statistic is reasonably stable for different pairs of languages $L_{i}, L_{j}$ with reasonably similar richnesses, comparisons between languages based on the $z_{i j}$ are likely to produce similar conclusions to those based on the $S_{i j}$.

\section{Anscombe's Method}

So far only pairwise comparison of languages has been considered. Anscombe proposed a method of analysis (Ross, pp. 51-53) which not only enables several languages to be considered simultaneously but is also supposed to take account of the interborrowing of words. The following quotation from Anscombe explains the rationale of the method. "- - the number of positive agreements - - - between two languages is not an entirely satisfactory measure of relationship by itself, since two languages not of recent common ancestry but which have borrowed extensively from other languages may have a large number of characters in common, while two small closelyrelated languages may have fewer characters in common. The richness of the languages should somehow be taken into account. The test devised below is for the null hypothesis that all the languages of the set are equally related, in such a way that any one is just as likely to have a particular — - character - as any other."

Anscombe's analysis is based on considering the distribution of quantities $u_{i j}$ where

$$
u_{i j}=r_{i j}-\frac{T_{i}+T_{j}}{l-2}+\frac{2 T}{(l-1)(l-2)},
$$

where $l=$ number of languages considered;

$$
\begin{aligned}
& T_{i}=\text { sum of all } r_{i j} \text { having one suffix equal to } i \\
& T=\text { sum of all the } r_{i j}, i<j
\end{aligned}
$$


'Thus the $u_{i j}$ are the $r_{i j}$ corrected for the richnesses of the languages $L_{i}$ and $L_{j}$. So a large $u_{i j}$ is supposed to imply high association. It is possible to test whether there is association among the family of $l$ languages as a whole. For Anscombe shows that, if there is equal association, a statistic proportional to the sum of the squares of all the $u_{i j}$ has approximately a chi-squared distribution with $1 / 2 l(l-3)$ degrees of freedom. Large values reject the hypothesis of equal association. Individual $u_{i j}$ can be examined to see which languages, if any, are associated.

There are objections to Anscombe's method. First, there is no reason to suppose that richness of the $r_{i j}$ should be corrected linearly by subtraction, as is done in obtaining the $u_{i j}$. However, it is also true to say that there is no particularly convincing reas on for arguing that the $r_{i j}$ are to be corrected multiplicatively as in Ellegård's measure $r_{i j} /\left(n_{i} n_{j}\right)^{1 / 2}$. Secondly, there is certainly some danger in correcting for richness on the supposition that it corrects for interborrowing. For suppose that two languages, $L_{i}$ and $L_{j}$, are not subject to interborrowing but have very different rates of decay of words. Then, starting from a common parent, they may well have attained different richnesses at a later period. In these circumstances there seems little to be said for reducing $r_{i j}$ as a measure of association because one language has a very slow rate of decay. The quantity $r_{i j}-$ or $r_{i j} / N$ - would seem to be as good a measure as any in the actual state of affairs, unless indeed the rates of decay themselves can be estimated. Unfortunately information for distinguishing between interborrowing and exceptional rate of decay is hardly ever available. If it can be assumed that there is no interborrowing, then Kendall's method can be used to estimate the rates of decay.

Graphical display methods and the construction of family trees

Suppose the measures of similarity between pairs of the languages $L_{1}, L_{2} \ldots L_{l}$ are given. It is required to construct a family tree linking similar languages and indicating the 
parent languages. If the number of languages is small, this can be done by inspection, though, of course, the judgment as to what size of similarity merits the linking of languages is almost entirely a subjective matter. If we invoke the concept of statistical significance in respect of the similarities, then it could well be suggested that languages not associated by a significant similarity should on no account be linked. In the case that the majority of the languages of a family are found to be mutually associated significantly, the problem of comparing the relative strength of significant similarities becomes dominant. If the number of languages is large, it is convenient to have an algorithm to do the linking automatically.

\section{(I) Single-link cluster analysis ${ }^{1}$}

Here the two languages with the largest similarity are linked first, then the two with the next largest similarity and so on, until a point is reached at which the similarities are so low that it is felt no longer desirable to join further languages to groups of languages already linked.

\section{Group-average cluster analysis}

A variant of this method consists in only joining a language to a group already linked if its »average" similarity with members of the group is the largest such average similarity out of those outside the group. "Average» can be defined in a number of ways, and a number of different methods of this type exist.

It should be emphasised that there is no such thing as a "best" clustering method, for different methods have their advantages and disadvantages depending on the structure of the data. Judgment of the results of tree construction is again a subjective matter.

¿ Cf. R. M. Cormack, "A review of classification», Journal of the Royal Statistical Society A. CXXXIV, 321-353. 


\section{(II) Mapping methods}

A similarity between languages may be in part a geographical matter; this would particularly be the case if the presence of words in the languages were due to interborrowing. And, quite apart from interborrowing, the evolution of languages could imply geographical association.

It may therefore be suggested that the similarities between languages could be used to construct coordinates for the languages in a space of a suitable number of dimensions - in the geographical case this number would be two - such that languages with high similarity would have coordinates close together in the space whereas dissimilar languages would be far apart.

In realisation, such methods are known as scaling methods, and two are in common use.

\section{Non-metric multidimensional scaling}

Given the $l$ languages $L_{1}, L_{2} \ldots L_{l}$, with coefficients of similarity $S_{i j}$, the data is represented in a coordinate space of $m$ dimensions by finding coordinates $\left\{x_{i \mathrm{~K}} ; i=1,2 \ldots l\right.$; $\mathrm{K}=1,2 \ldots m\}$ for each $L_{i}$ in a space with metric $d . m$ is to be as small as possible but, as far as possible, to satisfy the monotonicity condition

$$
d_{i j}<d_{k q} \text { when } S_{i j}>S_{k q} .
$$

That is, $L_{i}$ and $L_{j}$ should be nearer together than are $L_{k}$ and $L_{q}$ if $L_{i}$ and $L_{j}$ are more similar than $L_{k}$ and $L_{q}$. The choice of metric, $d$, is at our disposal, but is often taken to be the Euclidean distance. Various rules can be used to deal with "ties" $\left(S_{i j}=S_{k q}\right)$. Coefficients of stress can be defined to measure how well the monotonicity condition is satisfied in a given number of dimensions. The method is said to be nonmetric, because it depends directly only on the rank-ordering of the $S_{i j}$ (by reason of the condition $S_{i j}>S_{k q}$ ) and only indirectly on their absolute magnitudes. Further details of 
the method will be found in J. B. Kruskal, "Multidimensional scaling by optimising goodness of fit to a non-metric hypothesis", Psychometrika XXIX, 1-27.

Principal coordinates analysis

A similar method which uses the absolute magnitudes of the $S_{i j}$ more directly is that known by the above title. The theory underlying the method is essentially the following.

It is first necessary to define the similarity of a language with itself. This is not a problem for any of our similarity measures. For instance, the maximum value of the $z_{i j}$-statistic is $\sqrt{\mathrm{N}-1}$, and this is attained when the statistic is computed for the similarity of a language with itself. Next, a metric between the languages is defined by

$$
d^{2} i j=S_{i i}+S_{j j}-2 S_{i j}
$$

where $d_{i j}$ is the distance between $L_{i}$ and $L_{j}$. Now the $S_{i j}$ are corrected for mean similarities by forming the matrix $\alpha$ of elements

$$
a_{i j}=S_{i j}-\bar{S}_{i}+\bar{S}_{j}+\bar{S}
$$

where $\bar{S}_{i}$ is the row mean of the $l \times l S_{i j}$ matrix,

$\bar{S}_{j}$ is the column mean of the $S_{i j}$ matrix,

$\bar{S}$ is the average of all elements $S_{i j}$.

The latent roots $\lambda_{1}, \lambda_{2} \ldots \lambda_{l}$ and vectors of the matrix $\alpha$ are computed. The latent vectors are normalised so that the sums of the squares of the elements are equal to the corresponding latent roots. Finally suppose that the latent roots $\lambda_{1}, \lambda_{2} \ldots \lambda_{l}$ are ordered so that $\lambda_{1}>\lambda_{2}>\ldots>\lambda_{l}$.

'Then, in $l$-dimensional space, languages $L_{i}, L_{j}$ have coordinates given by the $i$, respectively $j$, elements of the $l$ latent rectors. The Euclidean distance between points with these coordinates is $d_{i j}$, so the representation of the distances between the languages by these points is a perfect one in $l$ dimensions. If we wish, we can seek to represent the languages in fewer than $l$ dimensions (say, $u$ ) by using just the first $u$ 
coordinates. Suppose the distance between $L_{i}$ and $L_{j}$ using. just the first $u$ coordinates is $d_{i j}^{(u)}$. Then we can assess the adequacy of using just $u$ dimensions to represent the data by the stress coefficient

$$
\sum_{i<j}\left\{\left[d_{i j}\right]^{2}-\left[d_{i j}^{(u)}\right]^{2}\right\} / \sum_{i<j}\left[d_{i j}\right]^{2}
$$

which can be computed as

$$
\sum_{i=1}^{u} \lambda_{i} / \sum_{i=1}^{l} \lambda_{i} .
$$

Further details of this method can be found in J. C. Gower, "Some distance properties of latent root and vector methods in multivariate analysis», Biometrika LIII, 325-338.

Again it is not possible to say which of the mapping methods will give the most meaningful results for a particular set of data. On the whole, the two methods gave similar results on the linguistic data on which they have been tried.

\section{THE URALIAN LANGUAGES}

In this section many of the techniques just discussed are applied to the Uralian languages. Of some techniques, the results are not reported here because they have given very similar results to those of others.

We have - naturally - taken the major division of the family, into Finno-Ugrian and Samoyede, as given. As far as the inventory of words is concerned, there is very little overlap between the two subdivisions.

\section{FINNO-UGRIAN}

For this subdivision six languages or language-groups ${ }^{1}$ were considered: Finnish $(\mathrm{F})$, as representing Baltic Fennic; Lappish (L); Mordvin (M); Cheremyss (C); Permian (P), that is Zyryene and Votyak; and Ugrian (U), that is, Vogul, Ostyak

1 In the context of these six - and only in this context - Language means 'language' or 'language-group'. 


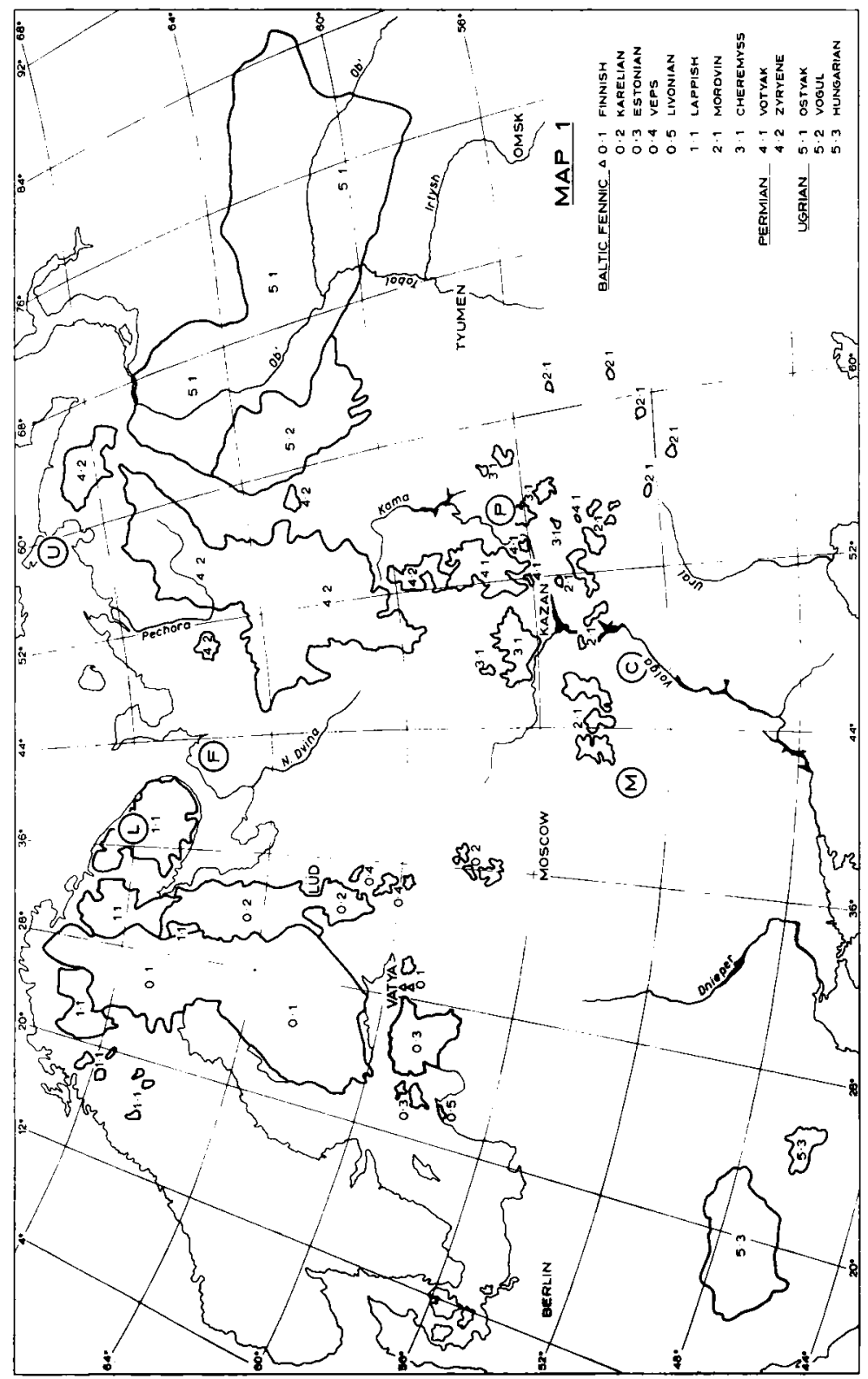


and Hungarian. It will be observed that we have not taken the obvious opportunity of joining up Mordvin and Cheremyss into one language-group -- they are undoubtedly closely-related, forming, as they do, the Volga Fennic group. We did this of set purpose, so as to leave a control: would our data and methods in fact reveal that these two languages are closely-related?

The inventory for this subdivision consisted of $N=965$ words. Table IV gives (1) the richnesses, $n_{i}$ (last column), (2) the number of words in common, $r_{i j}$ (above the diagonal), (3) the similarity coefficients $S_{i j}=r_{i j} /\left(n_{i}+n_{j}-r_{i j}\right)$ (below the diagonal). From the Table it is apparent that the similarity coefficients are all much the same, with most language-pairs having about $35 \%$ of the words in either in common. This would seem to suggest that the Finno-Ugrian languages constitute a family without very much close-relationship.

Map 1 shows the regions in which the various language-groups are located. A Principal Coordinates analysis of the $r_{i j}$ and $n_{i}$ was carried out to show the relative locations of the languages implied by these numbers. The first two coordinates were then plotted on the geographical map after suitable adjustment of the scale, the origin and the orientation of the principal coordinate axes. The stress-coefficient for a two-dimensional representation is high (48\%) and a three-dimensional system with stress $29 \%$ is better. However, the two-dimensional principal coordinates are very similar to those produced by the nonmetric scaling method, which has a stress of only $10 \%$. Thus the two-dimensional coordinates do provide a reasonable representation of the similarities implied by the $r_{i j}$. The principal coordinate points are shown on the map by the ringed languagegroup letters. If we leave Ugrian out of account, the closeness of these points to the corresponding geographical regions is striking. As Ugrian is composed of two regions far apart, the geographical centre has no meaning, and so a correspondence cannot be expected. We conclude that there is quite a strong relationship between language similarity and geographical proximity.

In Table $\mathrm{V}$, the values of the $z_{i j}$-statistic are given above the diagonal and the $u_{i j}$ obtained from Anscombe's analysis below it. The result of Anscombe's test was that the hypothesis 
TABLE IV

$\begin{array}{cccccccc} & \text { F } & \text { L } & \text { M } & \text { C } & \text { P } & \text { U } & \\ \text { F } & & 263 & 196 & 201 & 243 & 412 & 479 \\ \text { L } & 0.40 & & 165 & 176 & 213 & 352 & 451 \\ \text { M } & 0.33 & 0.28 & & 170 & 161 & 249 & 298 \\ \text { C } & 0.32 & 0.28 & 0.36 & & 197 & 295 & 341 \\ \text { P } & 0.35 & 0.31 & 0.27 & 0.33 & & 387 & 446 \\ \text { U } & 0.48 & 0.40 & 0.30 & 0.35 & 0.36 & & 786\end{array}$

that the languages are equally related, in the sense that any one is just as likely to have a particular word as any other one, is rejected at a very low probability level of 1 in 10000 . Of more interest are the $u_{i j}$ in their rôle as measures of association corrected for the richnesses of the languages. The languages most strongly associated are Mordvin and Cheremyss - our control is thereby vindicated — with Permian and Ugrian also strongly related. It was argued that a value of $z_{i f}$ much greater than 3 would provide strong statistically significant evidence of association on the grounds that, for the given richnesses of the two languages, there would be more words in common than would be expected by chance. Many of the language-pairs are covered by this rubric, with Mordvin and Cheremyss again strongly linked. But Lappish is not significantly related to either Permian or Ugrian by this argument. Principal coordinate maps of the $z_{i j}$ and $u_{i j}$ can be constructed in the same way as was the $r_{i j}$ map of Fig. 1 . As they are similar to this, and confirm the impression that strength of association is broadly related to geographical proximity, they are not reproduced here.

TABLE V

$\begin{array}{lrrrrrr} & \text { F } & \text { L } & \text { M } & \text { C } & \text { P } & \text { U } \\ \text { F } & & 5.1 & 6.7 & 4.3 & 2.8 & 3.6 \\ \text { L } & 10.0 & & 3.6 & 2.2 & 0.6 & -2.5 \\ \text { M } & 0.0 & 5.5 & & 9.4 & 3.2 & 1.1 \\ \text { C } & -19.5 & -8.0 & 43.0 & & 5.3 & 3.0 \\ \text { P } & -18.0 & -11.5 & -6.5 & 5.0 & & 3.9 \\ \text { IJ } & 27.5 & 4.0 & -42.0 & -20.5 & 31.0 & \end{array}$


The Finno-Ugrian family tree and its parameters

According to Kendall's model for the separation of two languages, $L_{i}$ and $L_{j}$, the parameters of the model are, first, $a_{i}$, the probability of survival of any root in $L_{i}$, along the line of descent to $L_{i}$ and, secondly, $S_{i j}$, the number of words in the common parent of $L_{i}$ and $L_{j}$.

The estimates of $S_{i j}$ for each pair of Finno-Ugrian languages are given in Table VI. Only one of the values of $S_{i j}$ is greater than the inventory size $N=965$, but many of the values are close to it. If Mordvin and Cheremyss are excluded it would not be unreasonable to postulate a parent language containing the 965 words from which the other languages are directly descended. (Of course it is profitless to speculate as to the number of words of the parent language which have disappeared in a 11 its descendants). The smaller figures for Mordvin and Cheremyss suggest that they had an intermediate parent from which several hundred of the original words had already disappeared.

Each pair of languages $L_{i}$ and $L_{j}$ provides estimates of $\alpha_{i}$ and $a_{j}$ along their lines of descent, and these are given in Table VII. Inspection of the rows of this table shows a high degree of stability in the estimates of the $\alpha$, with probabilities of survival for Finnish, Lappish and Permian of between 0.5 and 0.6. But Mordvin and Cheremyss retain about $55 \%$ of the words along their common line of descent but only $30 \%$ to $40 \%$ along their lines of descent with other languages. Thus one family tree suggested by these figures is that given in Fig. 1, which involves two parent languages $I$ and II and

TABLE VI

$\begin{array}{ccccccr} & \text { F } & \text { L } & \text { M } & \text { C } & \text { P } & \text { U } \\ \text { F } & & 821 & 728 & 813 & 879 & 914 \\ \text { L } & & & 814 & 874 & 944 & 1007 \\ \text { M } & & & & 598 & 825 & 941 \\ \text { C } & & & & & 772 & 909 \\ \text { P } & & & & & & 906 \\ \text { U } & & & & & & \end{array}$




\section{TABLE VII}

Language used with $L_{i}$ to derive $\alpha_{i}$

\section{Language}

$\begin{array}{lcccccc}L_{i} & \mathrm{~F} & \mathrm{~L} & \mathrm{M} & \mathrm{C} & \mathrm{P} & \mathrm{U} \\ \mathrm{F} & & 0.58 & 0.66 & 0.59 & 0.54 & 0.52 \\ \mathrm{~L} & 0.55 & & 0.55 & 0.52 & 0.48 & 0.45 \\ \mathrm{M} & 0.41 & 0.37 & & 0.50 & 0.36 & 0.32 \\ \mathrm{C} & 0.42 & 0.39 & 0.57 & & 0.44 & 0.37 \\ \mathrm{P} & 0.51 & 0.47 & 0.54 & 0.58 & & \\ \mathrm{U} & 0.86 & 0.78 & 0.83 & 0.86 & 0.87 & \end{array}$

seven probabilities or rates of survival labelled in an obvious notation $\alpha_{\mathrm{L}}, \alpha_{\mathrm{F}}, \alpha_{\mathrm{P}}, \alpha_{\mathrm{U}}, \alpha_{\mathrm{II}}, \alpha_{\mathrm{M}}, \alpha_{\mathrm{C}}$.

The statistical problem of how best to estimate the parameters from the data of the $r_{i j}$ and the $n_{i}$ is difficult. Some of the standard statistical techniques, such as maximum likelihood estimation, are intractable, because of the difficulty of writing down the marginal likelihood function for the data. The approximative technique employed can be briefly described as follows. It was assumed that Parent I contained all 965 of the inventory words. Then, for instance, the expected value of $r$ for Lappish and Finnish is $965 \alpha_{\mathrm{L}} \alpha_{\mathrm{F}}$. By taking logarithms, the expected value of $\log (r / 965)$ is approximately $\Theta_{\mathrm{L}}+\Theta_{\mathrm{F}}$, where $\Theta_{\mathrm{L}}$ and $\Theta_{\mathrm{F}}$ are $\log \alpha_{\mathrm{L}}$ and $\log \alpha_{\mathrm{F}}$ respectively. Thus the problem was changed to one involving a model linear in the $\Theta$ parameters; least squares techniques were used to estimate the $\Theta$, and hence the $a$. Further details of this approximative method of analysis will not be considered here, as the only purpose has been to show that there are values of the parameters which make the model fit the data quite well. In Table VIII the estimated parameter values and their approximate standard errors are given. These can be used to obtain the expected or predicted values of the model (e.g. $965 \alpha_{L} \alpha_{F}$ for $r_{L F}$ ) and approximate standard errors for these. The estimates of the $a$ are much as would be expected from Table VII, and the predicted values give quite a good approximation to the observed data. In fact the model fits the data better than could reasonably be expected when it is remembered that it assumes that all words have the same probability of survival and decay. 


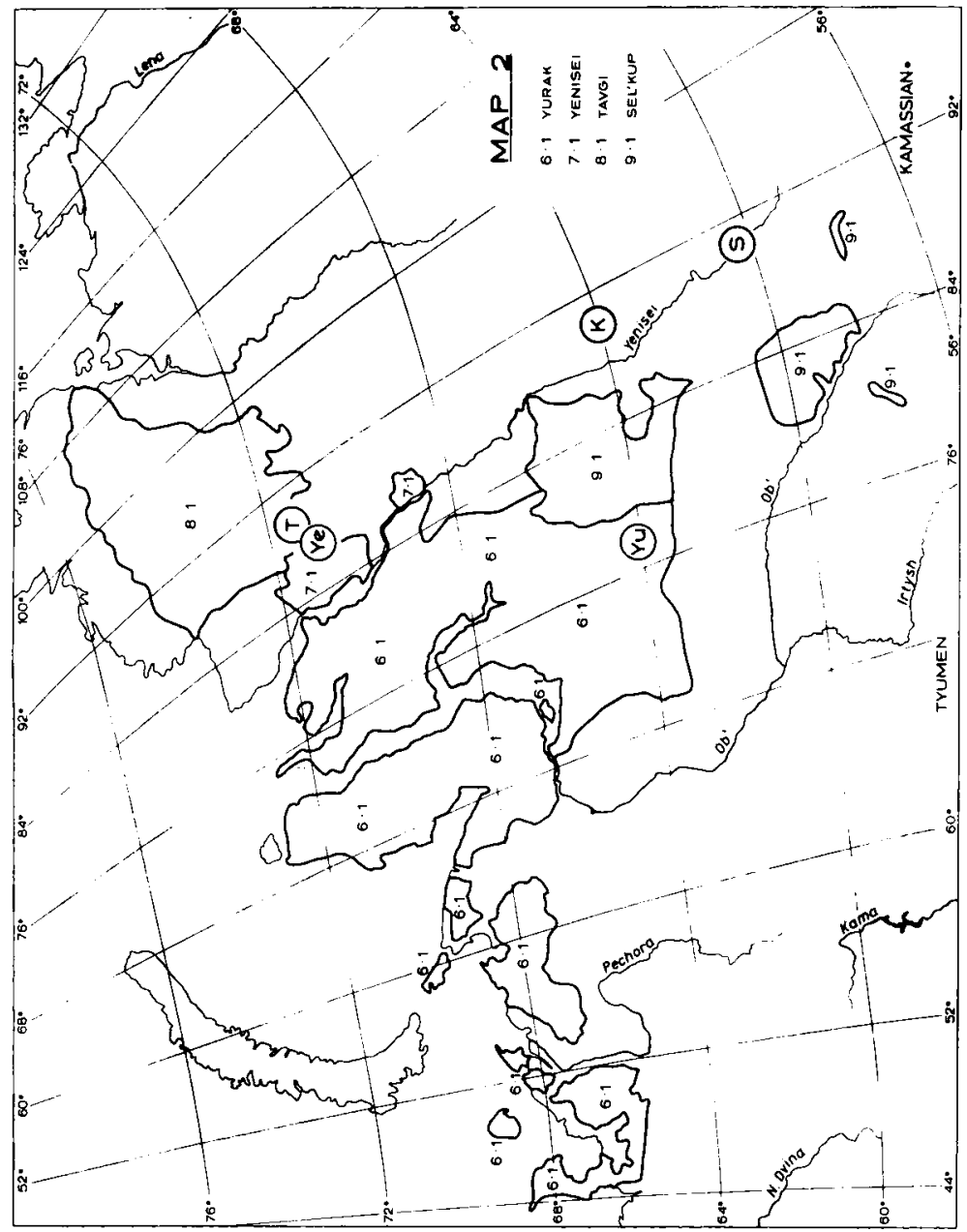


Figure 1.

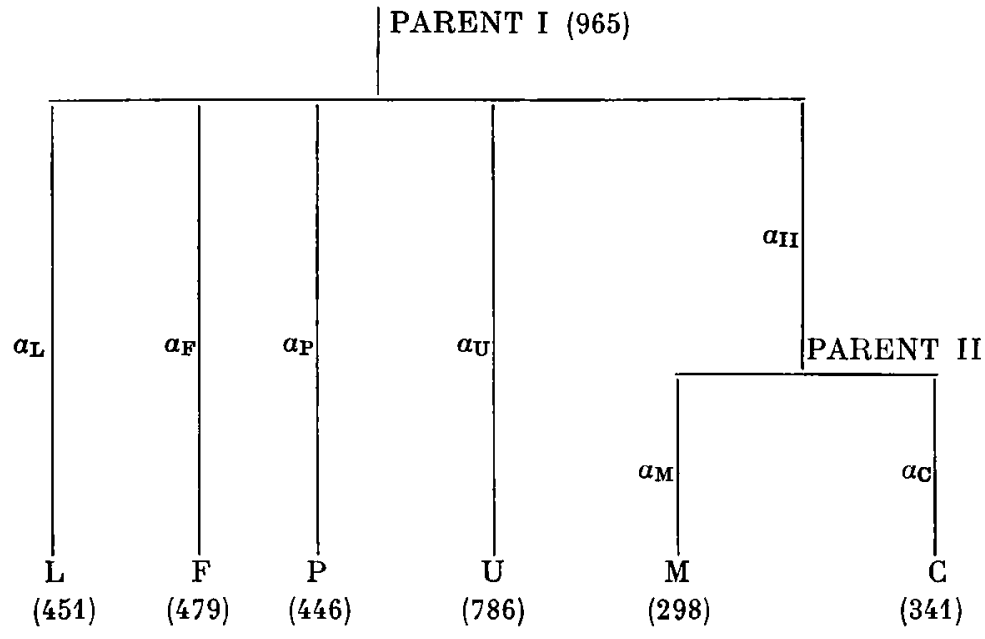

TABLE VIII

Estimates of the Finno-Ugrian $\alpha$ and predicted values for the $n_{i}$ and $r_{i j}$

$\begin{array}{ccccccc}\alpha_{\mathrm{L}} & \alpha_{\mathrm{F}} & \alpha_{\mathrm{P}} & \alpha_{\mathrm{U}} & \alpha_{\mathrm{II}} & \alpha_{\mathrm{M}} & \alpha_{\mathrm{C}} \\ 0.48 & 0.54 & 0.49 & 0.79 & 0.75 & 0.46 & 0.51 \\ (0.01) & (0.01) & (0.01) & (0.02) & (0.03) & (0.04) & (0.06)\end{array}$

\begin{tabular}{|c|c|c|c|c|c|c|c|}
\hline \multirow[b]{2}{*}{$n_{\mathrm{L}}$} & \multirow{2}{*}{$\begin{array}{c}\text { Observed } \\
451\end{array}$} & \multicolumn{2}{|c|}{ Predicted } & \multicolumn{2}{|c|}{ Observed } & \multicolumn{2}{|c|}{ Predicted } \\
\hline & & 460 & (13) & $r_{F P}$ & 243 & 254 & 1 \\
\hline$n_{\mathbf{F}}$ & 479 & 524 & (15) & $r_{\mathrm{FU}}$ & 412 & 414 & (1) \\
\hline$n_{\mathbf{P}}$ & 446 & 468 & (13) & $r_{F M}$ & 196 & 179 & \\
\hline$n_{\mathbf{U}}$ & 786 & 762 & (21) & $r_{F C}$ & 201 & 202 & \\
\hline$n_{\mathrm{M}}$ & 298 & $\mathbf{3 3 0}$ & $(10)$ & $r_{\mathrm{PU}}$ & 387 & 370 & (1 \\
\hline$n_{\mathrm{C}}$ & 341 & 371 & (11) & $r_{\mathbf{P M}}$ & 161 & 160 & \\
\hline$r_{\mathbf{L F}}$ & 263 & 250 & (9) & $r_{\mathrm{PC}}$ & 197 & 180 & \\
\hline$r_{\mathbf{L P}}$ & 213 & 223 & (8) & $r_{\mathrm{UM}}$ & 249 & 260 & (1 \\
\hline$r_{\mathrm{LU}}$ & 352 & 362 & (14) & $r_{\mathrm{UC}}$ & 295 & 293 & (1 \\
\hline LM & 165 & 157 & (6) & $r_{\mathrm{MC}}$ & 170 & 170 & \\
\hline C & 176 & 177 & (7) & & & & \\
\hline
\end{tabular}

The figures in brackets are approximate standard errors for estimates of parameters or predicted values. 


\section{SAMOYEDE}

For this subdivision five languages were considered: - Yurak (Yu); Tavgi (T); Yenisei (Ye); Sel'kup (S); Kamassian (K), as representing Sayan-Samoyede. The inventory for this subdivision consisted of $N=468$ words. Table IX is the counterpart of Table IV. Apart from implying a strong connection between Yenisei and Tavgi, the similarity coefficients are not very helpful, as they show a uniform picture of relationship with about $40 \%$ of the words in either of a language pair in common.

Map $2^{1}$ is the counterpart of Map 1. The principal coordinates analysis of the $r_{i j}$ in two dimensions gave a stress coefficient of about $30 \%$ compared with the non-metric scaling stress of about $2 \%$ but the language coordinates are similar in the two cases. As before, the points were plotted on the map and the resemblance of the map of language similarity to the geographical one is again quite striking. Table $\mathrm{X}$ is the counterpart of Table V. The hypothesis that the languages are equally related is rejected at a probability level of less than 1 in 10000 . Inspection of the table shows that the closest associations are between Yenisei and Tavgi, and then, between Sel'kup and Kamassian. The only $z$ that are not statistically significant are those between Yurak and Sel'kup or Kamassian. As in the case of Finno-Ugrian, a Principal Coordinate map of the $z$ shows a strong similarity to the actual geography.

\section{TABLE IX}

$\begin{array}{lcccccc} & \mathrm{Yu} & \mathrm{T} & \mathrm{Ye} & \mathrm{S} & \mathrm{K} & \\ \mathrm{Yu} & & 154 & 171 & 178 & 165 & 342 \\ \mathrm{~T} & 0.42 & & 149 & 126 & 118 & 180 \\ \mathrm{Ye} & 0.47 & 0.65 & & 135 & 125 & 196 \\ \mathrm{~S} & 0.42 & 0.40 & 0.42 & & 159 & 264 \\ \mathrm{~K} & 0.42 & 0.43 & 0.44 & 0.49 & & 215\end{array}$

${ }^{1}$ Having in mind what A. Joki, Kai Donners Kamassisches Wörterbuch (Lexica Societatis Fenno-ugricae VIII), p. XVIII, and, again, Die Lehnwörter des Sajansamojedischen (MSFOu 103), p. 27, has to say, we have entered Kamassian at Abalakova $\left(55^{\circ} 0 \mathbf{1}^{\prime} \mathrm{N}\right.$. $\times 94^{\circ} 50^{\prime} \mathrm{E}$.) $)$. 
The samoyede family tree and its parameters

Tables XI and XII are the counterparts of Tables VI and VII respectively. Only one of the values of $S_{i j}$ is greater than $N=468$.

The following points are of help in the devising of a family tree that might represent the data adequately. The Tavgi and Yenisei figure for $S_{i j}$ of $23 \bar{i}$ is the smallest, and, in view of their strong association, it is reasonable to postulate a parent from which about half the original inventory has been lost. This is supported by the $a_{i}$ figures which are very similar for the Tavgi and Yenisei rows and columns which show their similarity to other languages. The same kind of argument will justify a common parent for Sel'kup and Kamassian containing about 350 of the inventory words. Yurak, with its high proportion of words surviving, seems quite distinct from the other four languages. There arises the question whether the parent of Sel'kup and Kamassian could be the same as that of Tavgi and Yenisei. The difference in the estimated numbers of words in the parents suggests that this is not the case, and, so, trying to fit a family tree with the same immediate parent proves a failure. On the other hand, the entries in Tables XI and XII suggest that the parent of Sel'kup and Kamassian and that of Yurak and Tavgi must have been fairly closely related. After a certain amount of trial and error with different family trees having the properties described above, we concluded that the tree in Fig 2 was the one that fitted the data best. It involves three parent languages I, II and III, and seven rates of survival labelled in an obvious notation $\alpha_{\mathrm{Yu}}, \alpha_{\mathrm{II}}, \alpha_{\mathrm{S}}, \alpha_{\mathrm{K}}, \alpha_{\mathrm{III}}, \alpha_{\mathrm{T}}, \alpha_{\mathrm{Ye}}$. These parameters were estimated

TABLE $\mathrm{X}$

\begin{tabular}{lrrrrl} 
& Yu & \multicolumn{1}{c}{$\mathrm{T}$} & \multicolumn{1}{c}{$\mathrm{Ye}$} & \multicolumn{1}{c}{$\mathrm{S}$} & $\mathrm{K}$ \\
$\mathrm{Yu}$ & & 4.8 & 5.9 & -3.1 & 1.6 \\
$\mathrm{~T}$ & -4.3 & & 14.2 & 4.7 & 6.7 \\
$\mathrm{Ye}$ & 1.7 & 20.0 & & 4.6 & 6.6 \\
$\mathrm{~S}$ & 2.7 & -9.0 & -11.0 & & 7.0 \\
$\mathrm{~K}$ & 0.0 & -6.6 & -10.6 & 17.4 &
\end{tabular}


from the data in the same approximative way that was used for Finno-Ugriall, and the results are given in Table XIII, the counterpart of Table VIII. It is evident that the model produces predicted values which fit the observed data well, and all observed values are within one or two standard errors of the predicted values.

\section{CONCLUSIONS}

A number of different measures of association and methods of analysis have been tried with the Uralian data. It is not possible to say which method is - in any sense - the best, since they have different objectives, contribute different information, and rely on different assumptions. The construction of the family trees is undoubtedly the most ambitious part of the analysis. It is also the part which relies most heavily on simplifying assumptions and evolutionary models. Though the results apparently fit the data quite well, it would be foolish not to recognise that it is in this part of the analysis that we may be most seriously wrong. Mapping methods in respect of association measures seem particularly useful for this kind of data, for they show the extent to which language similarities are related to the geographical proximity of the languages. For the Uralian languages, this extent was substantial.

TABLE XI

$\begin{array}{lccccc} & \text { Yu } & \text { T } & \text { Ye } & \text { S } & \text { K } \\ \text { Yu } & & 400 & 392 & 507 & 446 \\ \text { T } & & & 237 & 377 & 328 \\ \text { Ye } & & & & 383 & 337 \\ \text { S } & & & & & 357\end{array}$

TABLE XII

$\begin{array}{lccccc} & \text { Yu } & \text { T } & \text { Ye } & \text { S } & \text { K } \\ \text { Yu } & & 0.85 & 0.87 & 0.67 & 0.77 \\ \text { T } & 0.45 & & 0.76 & 0.48 & 0.55 \\ \text { Ye } & 0.50 & 0.83 & & 0.51 & 0.58 \\ \text { S } & 0.52 & 0.70 & 0.69 & & 0.60 \\ \text { K } & 0.48 & 0.65 & 0.64 & 0.73 & \end{array}$


Figure 2.

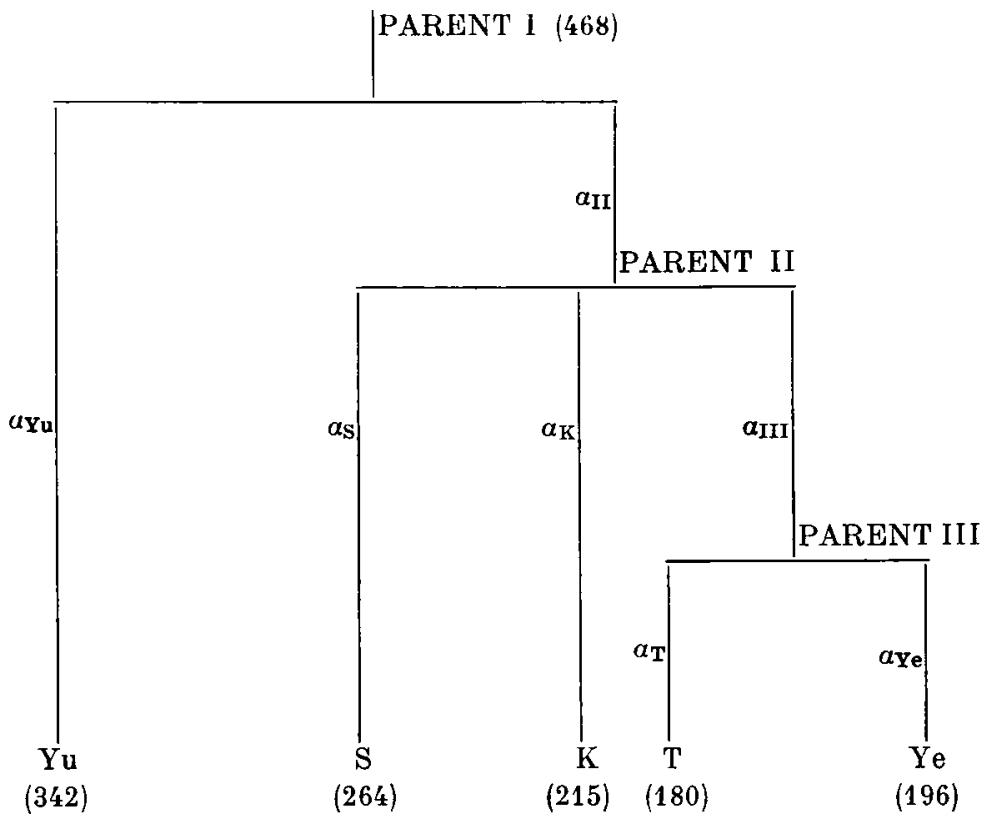

TABLE XIII

Estimates of the Samoyede $\alpha$ and predicted values for the $n_{i}$ and $r_{i j}$

$\begin{array}{ccccccc}\alpha_{\mathrm{Yu}} & \alpha_{\mathrm{II}} & \alpha_{\mathrm{S}} & \alpha_{\mathrm{K}} & \alpha_{\mathrm{III}} & \alpha_{\mathrm{T}} & \alpha_{\mathrm{Ye}} \\ 0.77 & 0.77 & 0.68 & 0.61 & 0.72 & 0.73 & 0.79 \\ (0.03) & (0.05) & (0.03) & (0.03) & (0.06) & (0.05) & (0.06)\end{array}$

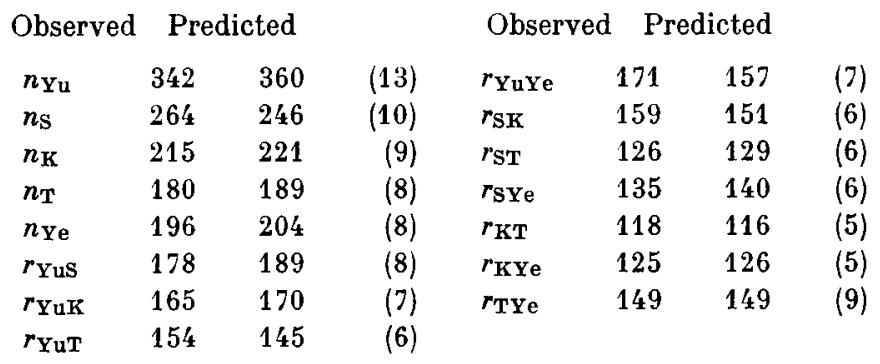

The figures in brackets are approximate standard errors for estimates of parameters or predicted values. 
The question of how the information from these two methods of mapping and family tree construction should be combined is a very difficult one. The family tree model is one which gives an implied temporal ordering to the concept of one language changing to another even if no time scale is attached to the branches. Mapping methods have been used here with some success to give information about how language changes have taken place spatially but we have not been able to infer any temporal ordering from these results. To combine the methods, a model for language change incorporating both geographical and temporal ideas is needed but we lack both such a model and the historical evidence by which it could be tested.

Paul Davies and Alan S. C. Ross 